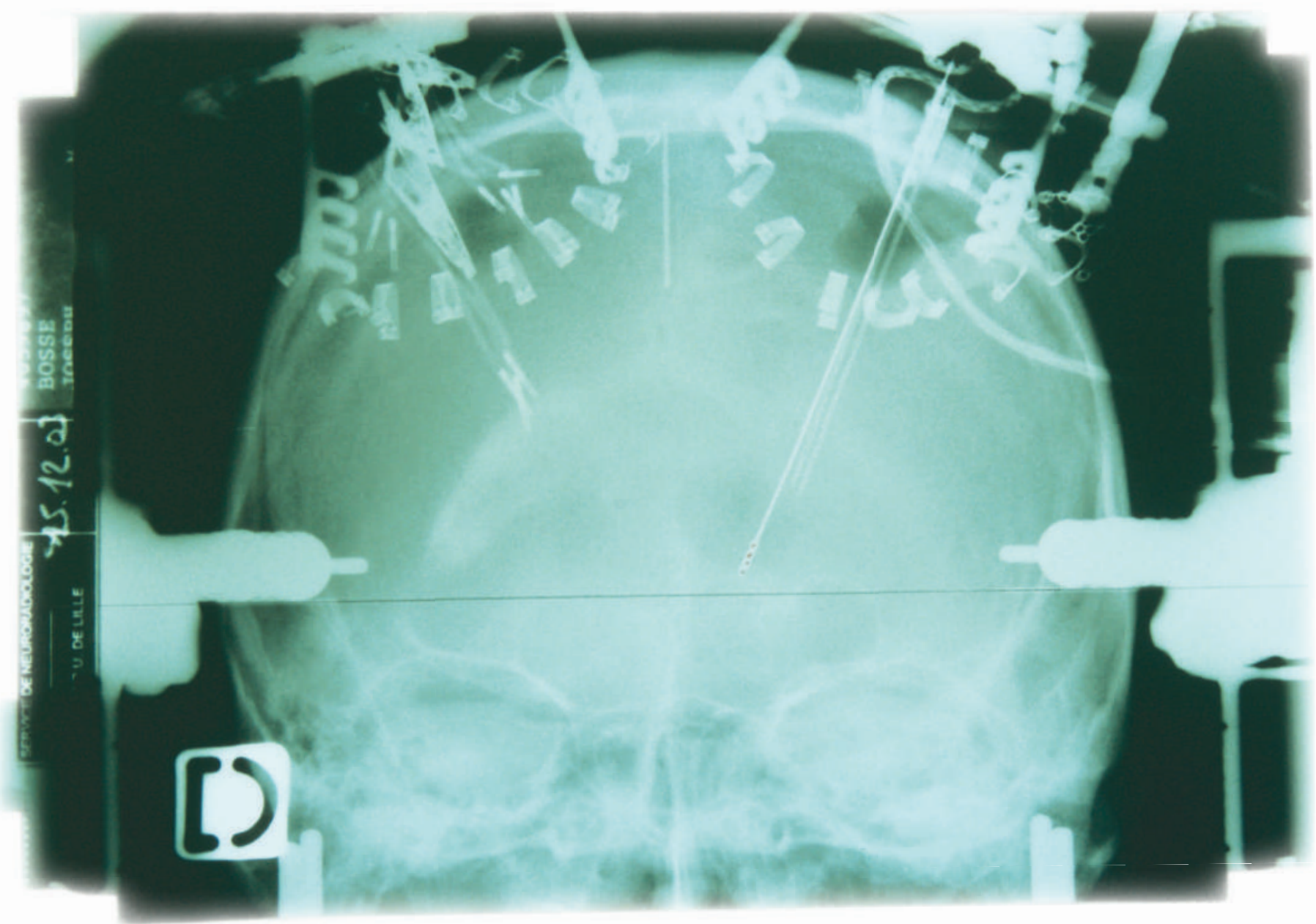

\title{
OPENING UP BRAIN SURGERY
}

\section{Neurosurgeons have unparalleled access to the human brain. Now they are teaming up with basic researchers to work out what makes it unique, finds Alison Abbott.}

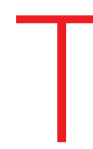

wo years ago, a top salesman in a major Italian engineering company drove himself to the emergency department of the San Matteo Hospital in Pavia. He was frightened. He could think clearly and he could move his hand normally, but for the past few days he hadn't been able to write. The business e-mails he had confidently typed out on his computer made no sense when he read them back. And he found he was unable to write a simple word by hand.

Tests at the hospital showed him to be apparently fit. He could express himself without problem, read, spell words out orally letter by letter - and he could even draw simple objects. The doctors started to wonder whether they should call in a psychiatrist. But when a brain scan showed what seemed to be a tiny tumour, they called neurosurgeon Lorenzo Magrassi instead. Magrassi immediately prescribed drugs to treat the swelling, which was pressing on surrounding brain areas, and within a couple of days the patient was able to write again.

Magrassi was intrigued. He had not heard of a similar case of agraphia - the inability to write. So when he operated the following week to remove the tumour, he also undertook a little research. The patient, who was in full agreement, was asked to speak while Magrassi stimulated his brain with an electrode. He was also provided with paper and a pen to jot down dictated sentences until the surgeon found just the spot that controlled writing.

Magrassi's techniques were not new: brain surgeons frequently stimulate around a tumour or other diseased tissue that they plan to remove while a patient is awake and able to answer questions. This 'functional brain mapping' allows them to identify the areas involved in speech and other functions that they would prefer not to cut into (see 'Open brain biography', page 868).

What is new is the way that neurosurgeons are using these techniques for bolder exploratory brain mapping, often in collaboration with basic researchers. In short, the surgeons have a unique opportunity to access and stimulate the human brain, and, as technologies improve, more of them are starting to use it. "Neurosurgery can contribute to neuroscience by giving a glimpse into the mind, a rare window into the brain," says surgeon Itzhak Fried from the University of California, Los Angeles. "We can start to look at uniquely human abilities."

Some of the collaborations springing up are tackling aspects of humanness - such as consciousness or language - that are of long-standing interest to neuroscientists and of practical relevance to neurosurgeons. Magrassi emphasizes that his
nas not purely experimental, but has application for future surgery. "It is often as clinically important to preserve writing after surgery as it is to preserve speech, and we need to understand which areas to avoid," he says.

Nonetheless, brain science has benefited. Over the next few months, and again with informed consent, Magrassi repeated his map- 
ping activity in two other patients with brain tumours even though they had not shown writing difficulties. He found that the area required for writing was always in the same spot in a structure called the superior parietal gyrus on the left side of the cortex. His results - yet to be published - extend theories about the neural circuitry of writing, a quintessentially human activity. Writing seems to co-opt brain structures in areas involved in language, visual processing and facilitating movement, and lesions in some of these areas can drastically interfere with writing ability. Normally these deficits are accompanied by others, such as an inability to speak, read or move normally. Since stimulation of the 'writing spot' did not interfere with these, Magrassi's studies suggest that the linguistic and motor circuits involved in writing are more integrated with each other than previously thought.

Exploratory brain studies are not yet commonplace. It can be difficult to experiment in the operating theatre with the active participation of patients, who are stressed and distracted.

But in some circumstances patients' brains can be available for days after the surgery. When neurosurgeons implant electrodes into the subcortical part of the brain to treat Parkinson's disease with deep-brain stimulation (DBS), they sometimes leave the leads outside the skull for a day or two to make sure that the procedure was successful before connecting them to a stimulator and battery and sewing everything under the skin.

In clinical trials of patients with psychiatric disorders, neurosurgeon Volker Sturm, at the University of Cologne, Germany, leaves the electrode leads hanging out even longer - up to four days - to provide time to experiment. Sturm is extending DBS treatment to disorders such as major depression, obsessivecompulsive disorder and alcoholism. The operation involves placing electrodes in a small area within the nucleus accumbens, a tiny structure that has a fundamental role in reward and is easily hijacked by addictive substances. Many of the tests that Sturm's neuroscience collaborators perform while the electrode leads are exposed have direct clinical relevance. Some, for example, are designed to investigate the effect of stimulation in the nucleus accumbens on neurons' electrophysiological properties and whether these properties can help to predict any psychiatric benefits that might later emerge.
Others look like experiments in basic brain science. In a study published last June ${ }^{1}$, Sturm and his collaborators investigated what happens in the brain when patients change strategies to fit new circumstances. A major function of the nucleus accumbens is to assess the value of a reward and adapt behaviour in response. The group analysed data from six patients fresh from surgery. A day after the electrodes were implanted in their left and right nuclei accumbens, the researchers recorded local field potentials around the electrodes. They also used electrodes placed on the scalp to record neurophysiological oscillations in the medial frontal cortex, which has direct connections to the nucleus accumbens.

The patients then did a simple gambling task in which they had to guess which side of

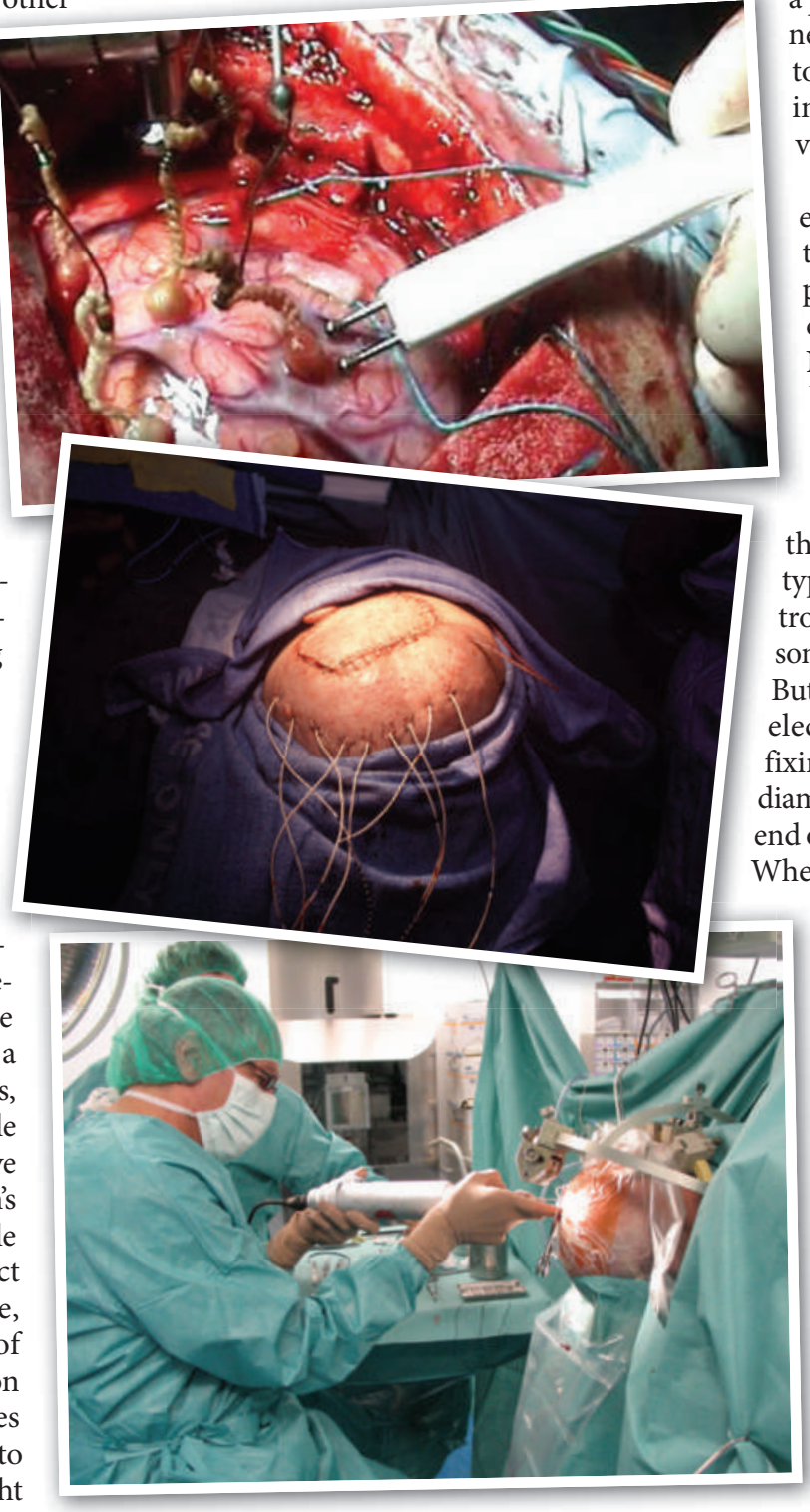

Brain surgery patients increasingly participate in research. a coin would pay out a cash prize. When the experimenters changed the odds of winning, the patients changed their gambling strategies - and the synchrony of electrical oscillations in the various brain areas also changed. The team concluded that a neural network between the nuclei accumbens and medial frontal cortex activates rapidly when the patients adjusted their behaviour to fit the new facts. "Yes, it is basic science," says Michael Cohen, a member of the collaboration and a psychologist at the University of Amsterdam, "but it may eventually have clinical relevance." For instance, he says, surgery might be avoided if the nucleus accumbens can be modulated indirectly via the medial frontal cortex, which can be stimulated through the skull.

New surgical procedures for epilepsy offer a particularly tantalizing opportunity for neuroscientists, because they allow access to the cortex - the region where thinking goes on - and the opportunity for very precise recording.

Some particularly brutal forms of epilepsy that don't respond to drug treatment originate in the medial temporal cortex and can be cured by surgical removal of the region responsible. Neurosurgeons identify the exact focus by implanting a handful of electrodes around the medial temporal cortex, waiting for the patients to have a spontaneous seizure and then determining the origin of the epileptic activity. They typically use 1-millimetre diameter electrodes that pick up the average signal from some one thousand to one million neurons. But more teams are starting to use 'microelectrodes' too. This technique involves fixing eight very fine recording wires, with diameters of less than 50 micrometres, to the end of the larger electrodes like wiry spiders. Whether the microelectrodes have further clinical benefit remains unknown, but it is not thought to do any harm, and for research it allows many levels of electrical signals to be recorded, from the oscillations in large populations of cells down to firing in single cells.

Fried has been using the electrode systems since the 1970 s and is the acknowledged pioneer of this technique in research. In the past halfdozen years, strides in data analysis have allowed faint and infrequent signals from single cells to be extracted from the noise. These are the sort of signals that excite those interested in human consciousness because they reflect rare or subtle events - such as recognizing the face of a celebrity. 


\section{OPEN BRAIN BIOGRAPHY}

Some milestones in invasive brain surgery and recording.
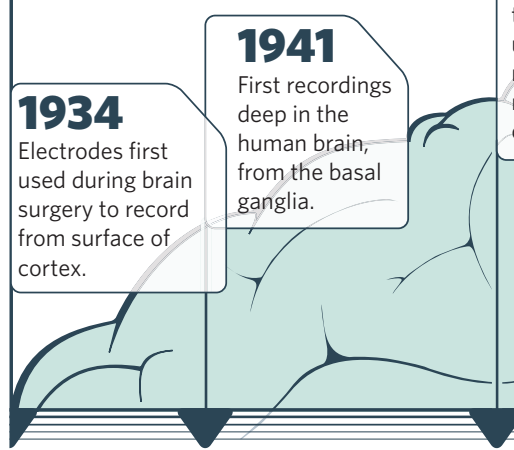

\section{8}

Wilder Penfield publishes The Excitable Cortex in Conscious Man, summarizing two decades of work using electrical recording to map the motor and sensory cortices

Rodrigo Quian Quiroga, a physicist-turnedneuroscientist and one of Fried's collaborators, hit headlines around the world in 2005 when he and his colleagues unveiled their concept of the 'Jennifer Anniston neuron'2. Quian Quiroga came to the California Institute of Technology (Caltech) in Pasadena as a postdoc in 2001 mainly for the opportunity of recording from single neurons in patients with epilepsy who were being operated on by Fried. He was particularly interested in how the human hippocampus, part of the medial temporal lobe, is involved in recognition of people or objects.

Recording from an array of 64 individual electrodes in a brain can generate hundreds of gigabytes of information a day. So Quian Quiroga made it his first task to find a better way to sort through the vast dumps of data. Having a background in physics helped, and by 2003 he had devised a neat algorithm that could sort through the tangled electrical signals and unambiguously identify spikes from single neurons. In one step he raised the entire game.

He tackled the recognition question by following the activity of single neurons while patients looked at hundreds of pictures on his laptop - from the actress Jennifer Aniston to the Sydney Opera House. Each neuron typically fired to one concept, but it did so rather flexibly: the Jennifer Aniston neuron would fire to different pictures of the actress but not to other celebrities. In some patients, Jennifer Aniston neurons would also fire to other actresses in Friends, the popular television series in which she starred. But they would never fire to other similar-looking, but otherwise unconnected, actresses. Quian Quiroga, now at the University of Leicester, UK, recalls his amazement when he found a single neuron in a patient firing in reaction to himself, even though they had met just a couple of days before.

He went on to show that these neurons fire only if the patients consciously recognize the pictures $^{3}$ and that they would also fire if a person was told the name of a person or object ${ }^{4}$.
"These neurons in the hippocampus encode information in a very abstract way, whatever type of sensory information leads to it," says Quian Quiroga. “This makes sense given that long-term memories are stored as abstractions - 'Jennifer Aniston as a concept' - and we tend not to remember details like what her hair looked like."

With these types of revelation emerging, it is perhaps not surprising that other epilepsy neurosurgeons are getting in on the act, starting to use microelectrodes and forge imaginative collaborations with neuroscientists, particularly now that the sophisticated components can be bought commercially.

It is a slow business though. One reason is the scarcity of patients who undergo this sort of neurosurgery. Even Fried's
"There is absolutely no risk for the patients, and they are just sitting around getting bored." - Adam Mamelak the experimenter can decide where in the brain to place the electrodes. In patients, electrodes must be positioned strictly according to clinical need. "The task of experimenters is to work out what we can do, given a particular distribution," says Quian Quiroga. "Patients always come first and we don't do anything with them if they are tired or have visitors."

All experiments are carefully regulated by ethics boards. Neurosurgeons say that patients nearly always agree to participate - and enjoy it. "There is absolutely no risk for them, and they are just sitting around getting bored," says Mamelak. "They take pride in contributing to the field of brain science," adds Fried.

Fried says that his collaborations with Qian Quiroga and others have allowed him to follarge centre operates on barely a dozen such people per year. Another is that it takes time to develop the expertise and build up a team. Neurologist Mark Richardson from King's College London, acknowledges that two years after implanting microelectrodes into his first patients, he is still very much at the beginning when it comes to research. "It takes a very long time for things to become routine," he says.

Neurosurgeon Adam Mamelak of the Cedars-Sinai Medical Center in Los Angeles, California, has built up collaborations with neuroscientists at Caltech. One such effort explored people's ability to detect 'novelty', something fundamental to many types of learning. The researchers identified some neurons in the hippocampus and amygdala that increase their rate of firing when the brain is confronted with new images, and some that do so when confronted with familiar images ${ }^{5}$. In another Caltech collaboration, he is looking at conscious decisionmaking in gambling tasks with neuroeconomist Antonio Rangel. "Animals can't perform these sorts of tasks," he says.

A big advantage of animals, though, is that low a line of research on memory. "Everything that you will consciously remember will have to be processed in the hippocampus," he says, adding that such studies could eventually help guide epilepsy surgery in the medial temporal lobe, "where memory networks sometimes overlap with epilepsy networks".

Fried says that some neurosurgeons wonder whether it would actually be unethical not to experiment, given how little is known about the brain entrusted to their hands, and given that the opportunity is there. The potential to learn about the neural basis of humanness - so that they can try to preserve it - is an

Alison Abbott is Nature's senior European correspondent.

1. Cohen, M. X. et al. J. Neurosci. 29, 7591-7598 (2009).

2. Quian Quiroga, R., Reddy, L., Kreiman, G., Koch, C. \& Fried, I. Nature 435, 1102-1107 (2005)

3. Quian Quiroga, R. Mukamel, R., Isham, E. A., Malach, R. \& Fried, I. Proc. Natl Acad. Sci. USA 105, 3599-3604 (2008).

4. Quian Quiroga, R., Kraskov, A., Koch, C. \& Fried, I. Curr. Biol. 19, 1308-1313 (2009).

5. Rutishauser, U., Mamelak, A. N. \& Schuman, E. M. Neuron 49, 805-813 (2006) opportunity that few want to pass up. 